patients with this often debilitating condition can be treated.

Castle, D. J., Deale, A., Marks, I. M., et al (1994) Obsessivecompulsive disorder: Prediction of outcome from behavioural psychotherapy. Acta Psychiatrica Scandinavica, 89, 393-398.

University of Western Australia

D.J. Castle Medical Research Foundation Building

Box X2213 GPO

Perth, WA 6001

\section{Cognitive therapy for panic}

SIR: We are grateful to Drs Clark et al (BJP, October 1994, 165, 557-559) for clarifying some points in their paper (BJP, June 1994, 164, 759 769). Their conclusions about cognitive therapy, however, remain problematic; their results can be explained non-cognitively just as well.

The Oxford cognitive therapy group's better outcome may reflect its cognitive therapy less than its getting firmer and more detailed exposure instructions (albeit in a cognitive cloak): attention to (a) stopping safety behaviours, which is a form of exposure; (b) behavioural experiments, which are a form of brief exposure; (c) interoceptive exposure; (d) diaries of behavioural experiments amounting to brief exposure (applied relaxation and imipramine patients were not asked to keep exposure diaries). Such elements constitute a good behavioural analysis and implementation.

The above procedures resemble Bandura's socalled mastery treatment which gave detailed teaching of exposure and reduced phobias. Clark $e t a l$ s cognitive therapy group improved without being asked to do prolonged exposure. They may have used an ingenious way to do brief effective exposure; this needs testing without cognitive components. A good test would be a comparison of their full cognitive-behavioural package with one containing only their behavioural elements and omitting cognitive elements such as their rationale, identification of misinterpretations of bodily sensations, challenging of evidence, and substitution of more realistic interpretations and restructuring images.

The authors say they did not aim to rule out the possibility that non-cognitive variables might also predict outcome, but rigorous ruling out is vital if cognitive theory is to be credible. Avoidance was not predictive. What about other non-cognitive variables such as Hamilton Anxiety, Beck Anxiety, general tension and anxiety, Beck Depression?
Change of beliefs may reflect general improvement rather than cause it. Moreover, a cognitive theory has to show that change in beliefs precedes improvement in other measures.

The Oxford group's non-severe cases "whose attacks were thought unlikely to be completely eliminated by situational exposure alone" were an easier-to-treat sample. Less severity (avoidance, anxiety, depression, disability, but not panic) predicts more response to various treatments (Basoglu et al, 1994a). Exposure to external cues may be unsuitable for the very few panic disorder patients who have neither avoidance nor situational panics, but such cases can respond to interoceptive exposure, which is not only "consistent with a cognitive theory of panic" but also with non-cognitive theories. The exclusion of severely agoraphobic cases remains puzzling. Despite most LondonToronto (LT) cases being severe, they were treated successfully in the clinic; very few were so housebound that they could not attend.

In most panic disorder patients, panic reduction is a weak yardstick unless disability is overcome too. Patients can become panic-free just by staying home. Unlike avoidance reduction, panic reduction relates little to lessening in work/social disability or global improvement (Basoglu et al, 1994b). Panic improved markedly with placebo, not only in the LT study (74\% after 8 weeks treatment, when the double-placebo dropout rate was only about $5 \%$ ) but also in the Upjohn Phase I and II multinational studies. The absence of non-pill groups in those studies does not vitiate this finding. The Oxford argument for spontaneous remission in panic in those studies seems unconvincing for patients who had been ill for a mean of 5-8 years and who with placebo improved more on panic than other ratings.

Clark et al also wonder if panic improved with placebo in LT patients because panics increased transiently after stopping drug before trial entry. This idea is disconfirmed by a fresh analysis. At week 0 , compared to patients who had had no prior medication, LT patients who stopped drug before week 0 had a similar number of situational and spontaneous panics, anticipatory anxiety, and illness severity. There was thus no overinflation in week 0 scores from recent drug withdrawal. At entry all LT patients had chronic panic disorder with agoraphobia by DSM-III criteria, having a mean of 5.2 major panics a week.

It remains unclear why the Oxford study used contrast groups with such a weak form of exposure (no exposure in weeks 1-4, no asking patients to stop safety signals and so stop avoiding, no note of 
patients being asked to do an hour's daily selfexposure and to keep homework diaries of this to enhance compliance), rather than the systematic exposure which usually does well in non-severe cases. Perhaps in such cases cognitive therapy enhances systematic exposure or helps even without it, but the Oxford design precludes conclusions on both these issues.

How much do exposure and cognitive therapy both work through teaching of (a) problem definition, goal setting, and recording of goal attainment, (b) habituation, (c) changing of beliefs, and (d) fostering of a sense of control? Such factors need not be mutually exclusive and could be synergistic, but need teasing out in firmer designs than those employed so far. Better definition is needed of which procedures are (1) uniquely 'cognitive', (2) uniquely 'behavioural', (3) overlap, or (4) are common to any treatment. We also need to separate therapy procedures (what the therapist does) from the theories on which they are said to be based. We agree with the authors that the treatments should be tried out in severe cases. We would be glad to work with them to clarify the minimum therapy procedures needed.

Basoglu, M., Marks, I. M., Swinson, R., et al (1994a) Pretreatment predictors of treatment outcome in panic disorder and agoraphobia treated with alprazolam and exposure. Journal of Affective Disorders, 30, 123-132.

Başoglu, M., MARKs, I. M., KILIc, C., et al (1994b) Relationship of panic, anticipatory anxiety, agoraphobia and global improvement in panic disorder with agoraphobia treated by alprazolam and exposure. British Journal of Psychiatry, 164, 647-652.

I. Marks

M. BASOGLU

H. NoshIRVANI

Institute of Psychiatry

Bethlem Royal \& Maudsley Hospital

London SE5 8AF

AUthors RePLy:

Our study (Clark et al, BJP, June 1994, 164, 759-769) showed that cognitive therapy is an effective treatment for panic disorder. Marks et al suggest that it is effective not because of its specific cognitive procedures but because it encourages exposure to feared stimuli. We excluded the possibility that situational exposure could account for the effects of cognitive therapy by arranging that the comparison psychological treatment (applied relaxation) included the same amount of exposure homework assignments as cognitive therapy. Neither of these treatments involved the use of exposure diaries. However, Marks et al suggest that exposure to interoceptive cues during the behav- ioural experiments that are part of cognitive therapy may be important, and call for a component analysis study. There are two such analyses (Margraf \& Schneider; Salkovskis et al) both of which were cited and discussed in our article. Together these studies indicate: first, that cognitive procedures are effective when used alone; and second, that combined interoceptive and situational exposure is also an effective anti-panic procedure. Further support for the efficacy of cognitive procedures alone comes from studies demonstrating their ability to block laboratory-induced panic (see Clark, 1993 for a review). It is less certain how effective is situational exposure alone. The LondonToronto (LT) study (Marks et al, 1993) did not find any difference between situational exposure alone and placebo/relaxation treatment as judged by panic measures. Marks et al (1993) argue that this failure to show a specific anti-panic effect for situational exposure was a consequence of the high response rate in their placebo/relaxation group. However, the end of treatment panic-free rate for applied relaxation in our study (50\%) was almost identical to the end of treatment ( 8 week) panic-free rate for the placebo/relaxation group in Marks $e t$ $a$ l's study $(47 \%)$ and we were still able to demonstrate a significantly higher response for cognitive therapy ( $90 \%$ panic-free).

Predicting long-term outcome. Cognitive theory predicts that patients who continue to misinterpret bodily sensations at the end of treatment should have a worse outcome at follow-up than patients who no longer make such misinterpretations. Correlational analyses supported this prediction. Marks et al wonder whether other non-cognitive variables might have been equally predictive. We have now investigated the variables suggested by Marks et al. None are significant predictors.

Severity of agoraphobic avoidance and response to cognitive therapy. We excluded from our study the 18 cases ( $20 \%$ of otherwise suitable referrals) who met DSM-III-R criteria for severe agoraphobic avoidance. Marks et al suggest that cognitive therapy would have done less well with these cases and that situational exposure would have been relatively more effective. Clearly a direct comparison between cognitive therapy and situational exposure with such patients is needed to address this question. However, existing data are not encouraging for their suggestion. First, patients in our sample had a considerable range of agoraphobic avoidance but initial level of avoidance did not predict response to either cognitive therapy or applied relaxation. Second, within our sample of 